\title{
Integrating highway alignment design capability to the Interactive Highway Safety Design Model (IHSDM): a two-lane highway case study
}

\author{
A. Maji ${ }^{1}$, M. K. Jha ${ }^{1} \&$ W. Kühn ${ }^{2}$ \\ ${ }^{1}$ Department of Civil Engineering, Morgan State University, USA \\ ${ }^{2}$ University of Leipzig, Germany
}

\begin{abstract}
Interactive Highway Safety Design Model (IHSDM) is a suite of software analysis and decision-support tools, widely used to check the design consistency in response to required safety and operational performance level along two-lane rural highways. It can only identify the inconsistent sections along a given highway. Therefore, after every check, the inconsistent sections are redesigned and checked again to ensure consistency. This process is manual and time consuming. The objective of this research is to incorporate the highway alignment design capability to the IHSDM. This will reduce the manual repetitive steps involved in the highway design and checking. In this study we have used highway geometric design properties and integrated it with IHSDM such that the inconsistent sections of the highway are replaced with sections having optimal safety and operational performance level. This method, first identifies the inconsistent sections from the given highway alignment using IHSDM and then replaces them with new, most appropriate sections. This process continues till all inconsistent sections are replaced. A real-world problem is solved in this paper using the proposed method.
\end{abstract}

Keywords: IHSDM, policy review, horizontal alignment, highway design, circular curve. 


\section{Introduction}

Transportation related injuries and deaths are unacceptably high in United States [1]. Therefore, safety is one of the critical issues in the field of highway design. A good design procedure should accompany the safety features with it. If it is not explicitly addressed during the design process then it may result in inconsistent or inappropriate design sections [2]. It is generally recommended that the design standards should exceed the minimum requirement to the maximum extent [3]. But limited resources and constraints due to right-of-way and environmental features often restrict highway designers in exceeding the minimum design standards [3]. It has been seen that designers do trade-off for curvature in horizontal alignment quite often [3]. The accident frequency is 1.5 to 4 times higher on curves compared to tangent sections of highway alignments [4]. It is also observed that accident frequency on rural highway curves is dependent on curvature along with length [3, 5], superelevation, lane width, shoulder width and presence of transition curve [3]. Therefore, horizontal circular curve is one of the important sections of the rural highway alignment from safety point of view. On the other hand, accident frequency on urban highways, where traffic volume is high and speed is low, is more dependent on intersections, access control and cross section features [5].

The above overview suggests that there is a need of a systematic approach that can allow the designer to consider the safety implications of design decisions, and the Integrated Highway Safety Design Model (IHSDM) serves that purpose [2]. While IHSDM is a great tool for identifying inconsistent sections, at present it has certain limitations. For example, it can't redesign the identified inconsistent sections to most suitable safe sections. This leads to repetitive, cumbersome and time consuming process of redesigning inconsistent highway alignment sections. The goal of this paper is to provide redesign capability to the IHSDM, so that the inconsistent sections are redesigned automatically after they are identified. Since horizontal curve is the single most important section of highway alignment that influence driver's behavior frequently leading to accidents [6], this work is focused on redesigning it to the maximum possible extent. In this research the attention is primarily directed to redesigning inconsistent sections along two-lane highways.

\section{IHSDM}

IHSDM is a suite of software developed for the Federal Highway Administration (FHWA) [7]. The main purpose of it is to evaluate safety and operational effects of highway alignment design on two-lane rural highways. The proposed model is applicable to both new design and redesign of an existing highway [2]. At present IHSDM can perform crash prediction, intersection review, policy review, traffic analysis, and check design consistency. An additional module about evaluation of driver operation under present design condition of highways is under development [7]. 
Generally, safety issues in highway design are reflected in the design policies and standards adopted by the design agencies [5]. Therefore, it is recommended that the highway design should be guided by the design policies developed. IHSDM can check the highway design sections' compliance to the highway geometric design policy in policy review module. It basically checks for the compliance of cross-section, horizontal alignment, vertical alignment, and sight distance information.

Since horizontal curves are the most critical sections of a highway alignment, special care should be taken in checking their design consistency. The IHSDM policy review module for horizontal alignments checks inconstancies in horizontal curves. It basically checks for consistency in design factors, such as the circular curve radius, ratio of composite curve, length of curve, and superelevation information in accordance with the policy standards. .

\section{Methodology}

A horizontal highway alignment basically comprises of tangent sections, circular curve sections, and transition curve sections that connect tangent and curve sections [8-10]. The orientation of these design sections are controlled by Point of Intersections (PI) of the alignment $[8,9]$. Therefore, the primary goal of this study is to modify the inconsistent design sections depending on the requirement without changing the PI. The procedure developed is represented through a flowchart in fig. 1 and discussed in subsequent paragraphs.

The IHSDM loads the alignment information for analysis from an input text file, which contains all horizontal and vertical alignment information. Then it performs consistency checks and gives the result as an output in an html file. The output file contains radius and length of circular curves information along with the minimum recommended value used for verification. From this output, radius and length of curvature information are separated and stored in a separate text file. In the proposed method these information along with the alignment information are used. The radius and length of circular curve sections, starting from the beginning of the alignment, are checked for consistency. The radius of curvature is checked before the length because the length affects the exposure of the curve $[11,12]$. If the radius of curvature is lesser than the minimum recommended value then it is increased to the required value or to the maximum possible value depending on the available length of the adjacent sections. Otherwise, the length is checked for adequacy. For inadequate length, the radius is increased as much as possible to satisfy the minimum length requirement. Even after finishing the procedure for inadequate radius, the length of curvature is checked and modified to the maximum extent possible. Each modification is followed by adjustment of the horizontal alignment information of all the sections. The process is repeated for all the circular curve sections of the given horizontal alignment.

The final modified horizontal alignment information is updated in the IHSDM input file. This file is now ready for re-analysis by IHSDM. The whole process described above is automated through a computer code, thus resulting in 
significant time savings in redesigning. This also adds the redesign capability to the IHSDM, which is lacking in the current version. The mathematical calculation behind the proposed methodology described above is explained in the subsequent section.

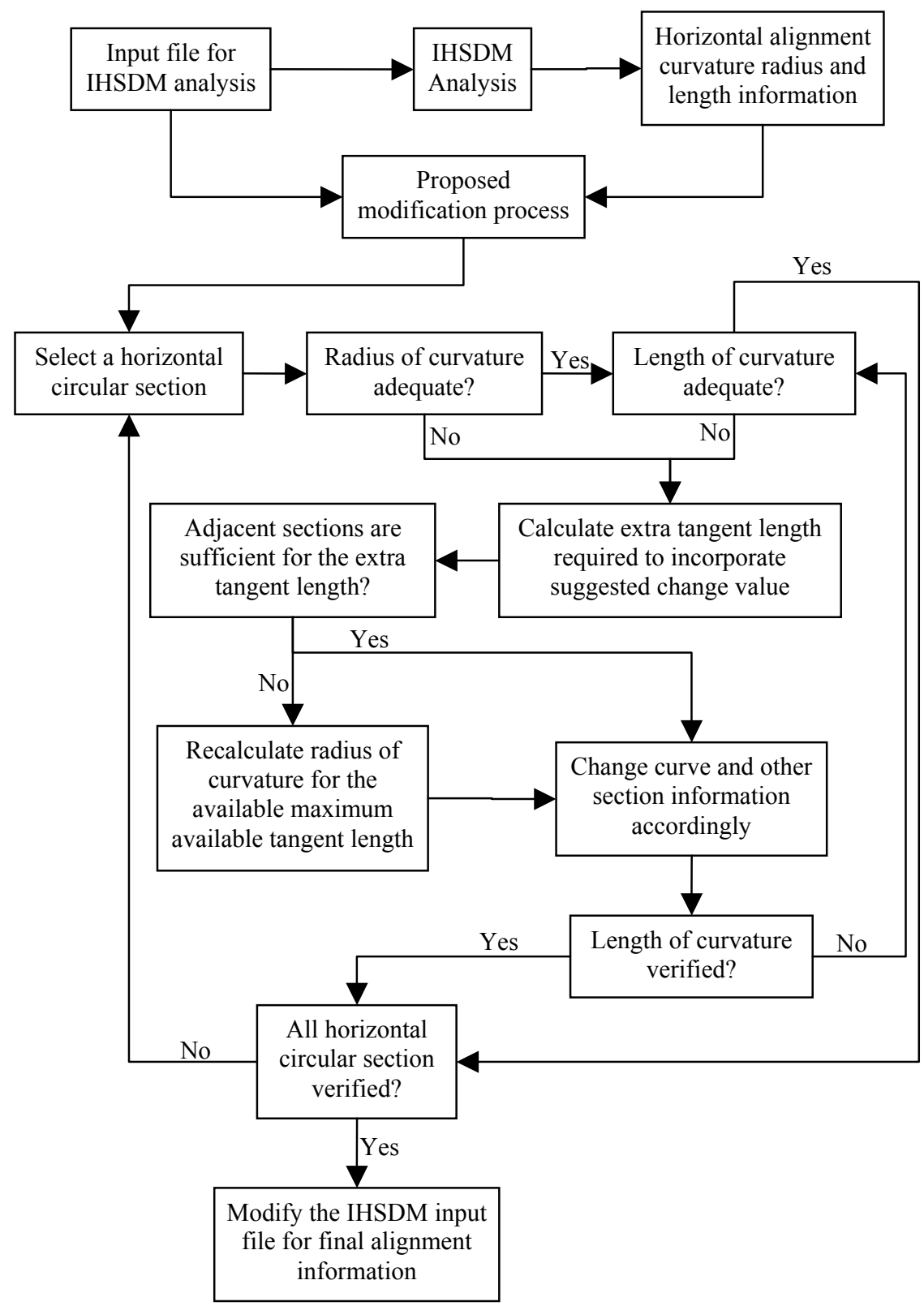

Figure 1: Flowchart of the proposed methodology. 


\section{Calculation procedure}

The calculation procedure adopted depends on the adequacy of the design radius and present length of curve, compared to required radius and length. All types of inconsistencies due to a combination of inadequate radius and length may arise. For example, radius and length both may not be adequate, or the radius may be inadequate but the length may be inadequate, or vice versa. The extent to which this inconsistency can be modified depends on the length of the adjacent tangent sections. Sometimes the tangent lengths may be adequate to incorporate the required changes but at other times it may not. The shorter of the adjacent tangent sections governs the redesigning procedure. Depending on the situation, three different cases of redesigning procedure is developed in this study. Before discussing the typical cases, let's describe the common calculations to be done at the beginning of the process.

First the center angle $(\Delta)$ and present tangent length $(T)$ of the circular curve section under consideration is calculated using eqns (1) and (2) respectively. Since we are not changing the PIs of the alignment, the center angle remains unaltered for all subsequent. Based on the policy recommended length of curve obtained through IHSDM analysis, the required radius $\left(R_{\text {len }}\right)$ of the curve is calculated using eqn (1) and compared with the policy recommended radius ( $R_{\text {rad }}$ ) obtained from the IHSDM analysis result. The maximum value of $R_{\text {len }}$ and $R_{\text {rad }}$ is considered as the required radius $\left(R_{r q d}\right)$ for the current analysis and verified with the design radius ( $R_{\text {des }}$ ). If $R_{\text {des }}$ is greater than the $R_{r q d}$ then the section is already safe and no further calculation is required, otherwise the tangent length required ( $T_{r q d}$ ) for $R_{r q d}$ is calculated using eqn. (2). The alignment is modified depending on the minimum available length of adjacent tangent sections $\left(L_{\text {tan }}\right)$. The calculation process for the three different modification cases are discussed in the following sub-sections.

$$
\begin{aligned}
\Delta & =\frac{360 L_{c i r}}{2 \pi R} \\
T & =R \tan \frac{\Delta}{2}
\end{aligned}
$$

where,

$\begin{array}{lll}\Delta & = & \text { Center angle } \\ L_{c i r} & = & \text { Length of the curve } \\ R & = & \text { Radius of curvature } \\ T & = & \text { Tangent length }\end{array}$




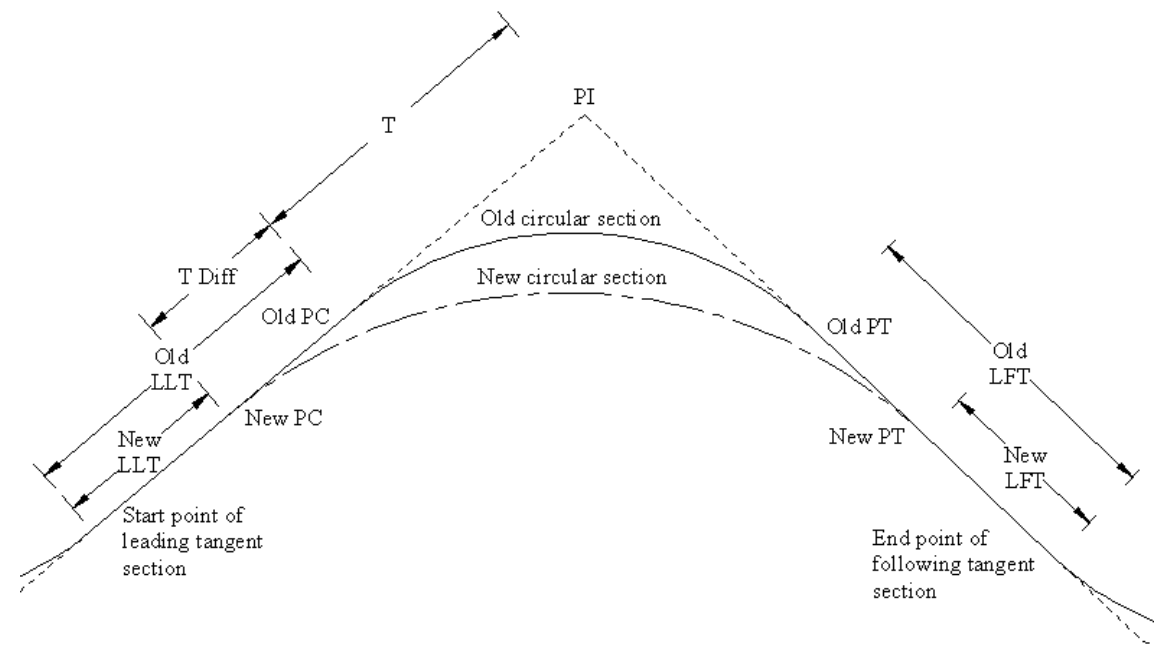

Figure 2: $\quad$ Both tangents are adequate.

\subsection{Case 1: adequate $L_{\tan }$}

This section describes the calculation procedure when $L_{\tan }$ is adequate to incorporate the required changes. It is the simplest procedure of the three cases discussed in this paper. The changed circular curve section fitted in the alignment will be similar to fig. 2 . In this case the calculation process starts with verifying the adequacy of $L_{\text {tan }}$ with the difference of $T_{r q d}$ and $T$. The difference is indicated in fig. 2 as $T$ Diff. The modified lengths of adjacent tangent sections are calculated next. The new length of leading tangent ( New LLT ) will be equal to the difference of old length of leading tangent (Old LLT) and T Diff. Similarly, the new length of following tangent ( New LFT ) will be equal to the difference of old length of following tangent (Old LFT) and T Diff. The $L_{\text {cir }}$ and new coordinate of the point of curvature (New $P C$ ) and point of tangency ( New PT) are changed accordingly from the old information (i.e., Old PC and Old PT ). If there is a transition curve inbetween the tangent sections and the circular curve section, then their starting and ending radius information is changed in compliance to the $R_{r q d}$. The change in length and coordinate information of the modified sections is passed to the subsequent sections and modified accordingly. After completing this process next circular curve information is selected and the process is started all over again. 


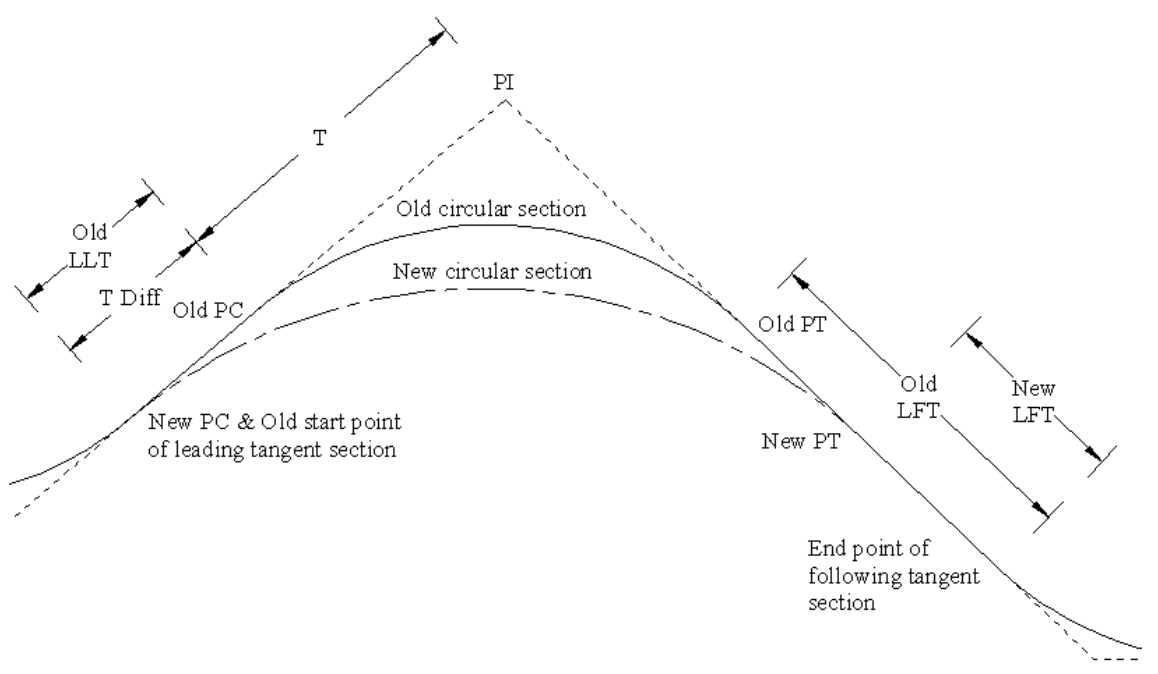

Figure 3: Inadequate leading tangent section.

\subsection{Case 2: length of leading tangent is inadequate}

This section describes the calculation procedure when length of the leading tangent (Old LLT) is inadequate to incorporate the required changes. The changed circular curve section fitted in the alignment will be similar to fig. 3. In this case the $T$ Diff is made equal to the Old LLT and the $T_{r q d}$ is recalculated by adding the $T$ with $O l d L L T$. Using the new $T_{r q d}$ value, $R_{r q d}$ is recalculated with the help of eqn (2) and then $L_{c i r}$ is calculated for the new $R_{r q d}$ using eqn (1). The leading tangent information is deleted from the alignment and the length of the following tangent (New LFT) is calculated by subtracting T Diff from Old LFT . The new coordinate of the point of curvature (New PC) and point of tangency (New PT) are changed accordingly as before. Transition curves, length and coordinate of modified curve sections are also changed as before. The changed information is passed to the subsequent sections and the coordinates are modified accordingly. Then, the next circular curve information is selected and the process is started all over again.

\subsection{Case 3: length of following tangent is inadequate}

This section describes the calculation procedure when length of following tangent (Old $L F T$ ) is inadequate to incorporate the required changes. The schematic change of a circular curve section is shown in fig. 4. This calculation procedure is similar to that discussed for Case 2 in section 4.2. The only 


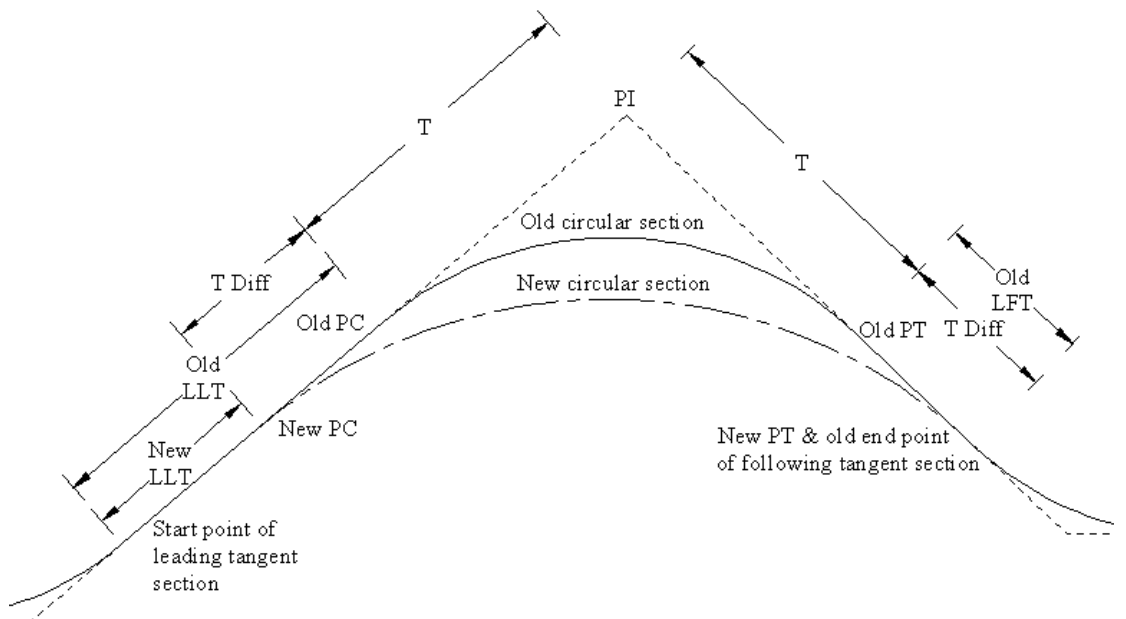

Figure 4: Inadequate following tangent section.

difference is that the T Diff is made equal to the Old LLT and the following tangent information is deleted instead of the leading tangent. The rest of the calculation procedure is the same as described in section 4.2.

\section{Case study}

We have considered a simple case study of a sample highway section to verify our developed methodology. The horizontal alignment of the sample highway section consists of three tangent sections and three simple circular curve sections. Details of the alignment are presented in table 1. This section was analyzed by IHSDM considering American Association of Sate Highway and Transportation Officials (AASHTO) Green-Book policy [13] for a speed of 80 $\mathrm{kmph}$. The recommendation for radius and length of the circular curve section is also noted in table 1 .

Table 1: Horizontal alignment before modification.

\begin{tabular}{|c|c|c|c|c|c|c|c|}
\hline $\begin{array}{c}\text { Start } \\
\text { station }\end{array}$ & $\begin{array}{c}\text { End } \\
\text { station }\end{array}$ & $\begin{array}{c}\text { Section } \\
\text { type }\end{array}$ & $\begin{array}{c}\text { Section } \\
\text { length }\end{array}$ & Radius & \multirow{2}{*}{$\begin{array}{c}\text { Curve } \\
\text { direction }\end{array}$} & \multicolumn{2}{|c|}{ Recommendation } \\
\hline 0.000 & 10.000 & Tangent & 10.000 & - & - & - & - \\
\hline 10.000 & 83.304 & $\begin{array}{c}\text { Circular } \\
\text { Curve }\end{array}$ & 73.304 & 200.0 & Left & 230 & 240.00 \\
\hline 83.304 & 183.304 & Tangent & 100.000 & - & - & - & - \\
\hline 183.304 & 352.208 & $\begin{array}{c}\text { Circular } \\
\text { Curve }\end{array}$ & 168.904 & 395.0 & Right & 230 & 240.00 \\
\hline 352.208 & 502.210 & Tangent & 150.002 & - & - & - & - \\
\hline 502.210 & 752.210 & $\begin{array}{c}\text { Circular } \\
\text { Curve }\end{array}$ & 250.000 & 275.0 & Right & 230 & 240.00 \\
\hline
\end{tabular}


It is observed from table 1 that the first circular curve section is inconsistent with respect to both radius and length, the second circular curve section is inadequate with respect to the recommended length only, and the third circular curve is designed well above the recommended values. Therefore, the sample horizontal alignment, recommended radius and length information are loaded to the computer program specifically developed for this purpose. This program modified the alignment information in accordance with the developed methodology and calculation procedure discussed earlier. The new alignment information after modification is shown in table 2.

Table 2: Horizontal alignment after modification.

\begin{tabular}{|c|c|c|c|c|c|}
\hline $\begin{array}{c}\text { Start } \\
\text { station }\end{array}$ & $\begin{array}{c}\text { End } \\
\text { station }\end{array}$ & $\begin{array}{c}\text { Section } \\
\text { type }\end{array}$ & $\begin{array}{c}\text { Section } \\
\text { length }\end{array}$ & Radius & $\begin{array}{c}\text { Curve } \\
\text { direction }\end{array}$ \\
\hline 0.000 & 93.080 & $\begin{array}{c}\text { Circular } \\
\text { Curve }\end{array}$ & 93.080 & 254.0 & Left \\
\hline 93.080 & 146.980 & Tangent & 53.900 & - & - \\
\hline 146.980 & 386.980 & $\begin{array}{c}\text { Circular } \\
\text { Curve }\end{array}$ & 240.000 & 561.3 & Right \\
\hline 386.980 & 500.882 & Tangent & 113.902 & - & - \\
\hline 500.882 & 750.882 & $\begin{array}{c}\text { Circular } \\
\text { Curve }\end{array}$ & 250.000 & 275.0 & Right \\
\hline
\end{tabular}

After carefully analyzing the final alignment information it is observed that the first tangent section is replaced and the first circular curve section is stretched to the maximum, to satisfy both recommended radius and length to the extent possible. The second circular curve section is also modified to satisfy the length inadequacy. But, the third circular curve remains the same as expected; only the station information is changed to adjust the changes of the previous sections. The remaining tangent sections are adjusted accordingly.

\section{Conclusion}

The computer code developed based on the methodology proposed in this paper works well and gives designer an opportunity to modify the inconsistent sections identified by the IHSDM analysis. At present, the code is not integrated with the available IHSDM software suite but it can be done if the source code for IHSDM was available. This integration will not only help the designer with the modification of highway alignment design but will also give IHSDM an alignment design capability. Since IHSDM performs analysis for two-lane rural highways, the proposed method is only applicable to two lanes. However, the developed methodology can easily be extended to multi-lane highways. Moreover, the methodology is only good for horizontal alignments since at present it does not consider the effects of vertical alignments. Minimization associated to redesign and other costs are also not considered in this study. While these enhancements can be made in future works, the present work can be considered as the first step to integrate highway design capability to the IHSDM. 


\section{Acknowledgement}

This work was completed at the Center for Advanced Transportation and Infrastructure Engineering Research (CATIER; www.eng.morgan.edu/ catier) at the Morgan State University.

\section{References}

[1] Safety Saves, http://tti.tamu.edu/researcher/v40n3/40_3.pdf

[2] Paniati, J.F. \& True, J., Interactive highway safety design model (IHSDM): designing highways with safety in mind. Transportation Research Board, Transportation Research Circular, 453, pp. 55-60, 1996.

[3] McGee, H.W., Hughes, W.E. \& Daily, K., Effect of highway standards on safety. Transportation Research Board, National Cooperative Highway Research Program Report, 375, 1995.

[4] Zager, C., Twomey, J., Heckman, M. \& Hayward, J., Safety effectiveness of highway design features volume II, alignment. Federal Highway Administration, Report No. FHWA-RD-91-045, Washington D.C., 1992.

[5] Pfefer, R.C., Neuman, T.R. \& Ranb, R.A., Improved safety information to support highway design. Transportation Research Board, National Cooperative Highway Research Program Report, 430, 1999.

[6] Krammes, R.A., Brackett, R.Q., Shafer, M.A., Oheson, J.L., Anderson, I.B., Fink, K.L., Collins, K.M., Pendleton, O.J. \& Messer, C.J., Horizontal alignment design consistency for rural two-lane highways. Federal Highway Administration, Report No. FHWA-RD-94-034, Washington D.C., 1995.

[7] Interactive Highway Safety Design Model (IHSDM), http://www.tfhrc.gov/safety/ihsdm/ihsdm.htm

[8] Kim, E., Jha, M.K. \& Son, B., Improving the Computational Efficiency of Highway Alignment Optimization Models through a Stepwise Genetic Algorithms Approach. Transportation Research, Part B, 39(4), pp. 339-360, 2005.

[9] Jha, M.K. \& Schonfeld, P., A Highway Alignment Optimization Model using Geographic Information Systems. Transportation Research, Part A, 38(6), pp. 455-481, 2004.

[10] Kim, E., Jha, M.K., Lovell, D.J. \& Schonfeld, P., Intersection Modeling for Highway Alignment Optimization. Computer-Aided Civil and Infrastructure Engineering, 19(2), pp. 136-146, 2004.

[11] Glennon, J.C., An evaluation of design criteria for operating trucks safely on grades. Highway Research Board, Highway Research Record, 312, 1970.

[12] Solomon, D., Accidents on main rural highway related to speed, driver and vehicle. Federal Highway Administration, Washington D.C., 1970.

[13] A Policy on Geometric Design of Highways and Streets. American Association of State Highway and Transportation Officials (AASHTO), Washington, DC, 2001. 